eISSN 2444-7986

DOI: https://doi.org/10.14201/orl201674.14643

Artículo de revisión

\title{
UTILIDAD CLÍNICA DE LOS POTENCIALES EVOCADOS MIOGÉNICOS VESTIBULARES (VEMPS)
}

\section{Clinical utility of the Vestibular-Evoked-Myogenic-Potentials (VEMPs)}

\author{
José Ignacio BENITO-OREJAS
}

SACYL. Hospital Clínico Universitario de Valladolid. Servicio de Otorrinolaringología y Patología cervicofacial. Valladolid. España.

Correspondencia: jibenito@ono.com

Fecha de recepción: 29 de mayo de 2016

Fecha de aceptación: 15 de junio de 2016

Fecha de Publicación: 18 de junio de 2016

Conflicto de intereses: Los autores declaran no tener conflictos de intereses

Imágenes: Los autores declaran haber obtenido las imágenes con el permiso de los pacientes

Política de derechos y autoarchivo: se permite el autoarchivo de la versión post-print (SHERPA/RoMEO)

Licencia CC BY-NC-ND. Licencia Creative Commons Atribución-NoComercial-SinDerivar 4.0 Internacional

๔ Universidad de Salamanca. Su comercialización está sujeta al permiso del editor

RESUMEN Introducción y objetivo: A principios de los años 90, Colebath y Halmagyi demuestran que es posible medir el potencial miogénico evocado del músculo esternocleidomastoideo a partir de una estimulación acústica o vibratoria del laberinto, dando nombre a los que desde entonces denominamos «potenciales evocados miogénicos vestibulares» (VEMPs: Vestibular-EvokedMyogenic-Potentials). El objetivo de esta revisión consistirá en exponer las bases anatomofuncionales de los VEMPs y describir sus aplicaciones clínicas actuales. Método: Se trata de un trabajo descriptivo donde, a partir de la bibliografía que consideramos más destacada, se resumen las características, procedimientos de obtención y utilidad clínica de los VEMPs. Resultados y conclusiones: Mediante el registro de VEMPs oculares (oVEMPs) y cervicales (cVEMPs), podemos obtener de forma práctica, información clínica sobre las funciones utricular y sacular. Ambos procedimientos son específicos, sencillos, rápidos, reproducibles y seguros. Cuando estos resultados se combinan con otras pruebas vestibulares, se consigue llegar a tener un conocimiento funcional de cada uno de los receptores sensoriales del laberinto.

PALABRAS CLAVE potenciales evocados miogénicos vestibulares; sáculo y utrículo; membrana otolítica; sonido; vibración; enfermedades del laberinto

SUMMARY

Introduction and objectives: At the beginning of the 1990s, Colebath and Halmagyi demonstrated that measuring the potential myogenic evoked from an acoustic or vibratory stimulation of the labyrinth is possible, giving name to what since then we call Vestibular-Evoked-MyogenicPotentials (VEMPs). The objective of this review, aims to meet the anatomic-functional bases of the VEMPs and to describe their current clinical applications. Method: This is a descriptive work from the most relevant bibliography, summarizing the features, obtaining procedures and clinical utility of the VEMPs. Results and conclusion: Using the ocular VEMPs (oVEMPs) and 
cervical (cVEMPs), we can obtain practical, clinical information on the utricular and saccular functions. Both procedures are specific, simple, fast, reproducible and safe. When these results are combined with other vestibular tests, they provide knowledge of the functional of each one of the sensory receptors of the labyrinth.

KEYWORDS

vestibular evoked myogenic potentials; saccule and utricle; otolithic membrane; sound; vibration; labyrinth diseases

INTRODUCCIÓN ANATOMOFISIOLÓGICA AL SISTEMA VESTIBULAR

El sistema vestibular representa el giroscopio del cuerpo humano [1], que a través de un funcionamiento permanente, proporciona al cerebro información sobre el movimiento de la cabeza y su orientación con respecto a la gravedad. El mecanismo responsable radica en la inclinación de unos cilios muy sensibles que se encuentran sobre las células ciliadas del laberinto (específicamente en las crestas de los 3 conductos semicirculares (CS) y en las 2 máculas de cada oído). Estas estructuras sensoriales están albergadas en el interior del laberinto membranoso, relleno de endolinfa y en continuidad con el líquido cefalorraquídeo a través del acueducto coclear [2].

Los CS están estructuralmente especializados para transducir la aceleración angular (rotación de la cabeza) y las máculas, para responder a la aceleración lineal producida por los movimientos de la cabeza (translación de uno a otro lado, de arriba hacia abajo o de delante hacia atrás) o a su inclinación (cambio de orientación). Cada célula vestibular tiene un kinocilio y 40-70 esterocilios; cuando los esterocilios se inclinan hacia el kinocilio, la célula se despolariza, incrementándose la tasa de descarga de sus células ganglionares y cuando se inclinan en dirección contraria al kinocilio, se hiperpolariza y disminuye la actividad de descarga de las neuronas aferentes. Los tres CS, angulados $90^{\circ}$ unos de otros, permiten obtener información de la aceleración angular en las tres dimensiones del espacio. De manera similar, las dos máculas, mantienen una angulación de $90^{\circ}$; estando la utricular próxima al plano horizontal y la sacular al vertical, por lo que el sistema vestibular capta también información de las aceleraciones lineales en cualquier dirección. Cada movimiento natural de la cabeza genera aceleración lineal y angular, de forma que la función vestibular normal trabaja integrando continuamente las respuestas de los CS y otolitos [3]. Las señales recibidas en el laberinto, llegan al núcleo vestibular del tronco del encéfalo (TE) a través de los axones de las células del ganglio de Scarpa que se encuentran formando el nervio vestibular. EI CS superior (CSS), el lateral u horizontal (CSH) y el utrículo, son inervados por el nervio vestibular superior y el CS posterior (CSP) y el sáculo por el nervio vestibular inferior. La función vestibular (al contrario de la coclear) no es "consciente» hasta que algo funciona mal, respondiendo entonces con una sintomatología incapacitante [2].

Las máculas se denominan por el lugar que ocupan dentro del laberinto membranoso, el utrículo contiene la mácula utricular y el sáculo, la sacular. Están constituidas por una densa capa de cristales de carbonato cálcico, las otoconias, empotradas en el área superficial de la membrana otolítica. En la parte interna de esta membrana, los cilios de las células ciliadas se proyectan sobre la misma. La capa otolítica tiene la misma densidad que la endolinfa, pero la de las otoconias es 3 veces superior. Ambas máculas presentan una estructura similar y responden al mismo principio físico. Cualquier fuerza lineal va a trasladar la capa otolítica, que se desliza a través de la superficie macular, desplazando los cilios de las células receptoras y produciendo un cambio de potencial en la membrana de estas células. Hay alrededor de 33.000 células receptoras en la mácula utricular y 19.000 en la sacular; y a diferencia del sistema auditivo se produce una convergencia hacia las fibras sensoriales aferentes, de manera que unas 6.000 neuronas salen del utrículo y otras 4.000 del sáculo [2].

Como hemos explicado, cada célula receptora está polarizada, es decir, responde mucho más en una dirección (aquella en la que los esterocilios se inclinan hacia el kinocilio) que en la contraria. La dirección óptima determina el vector de polarización celular. Hay un perfecto ordenamiento celular en cada mácula en relación a una línea central denominada estriola. En la mácula utricular, los vectores de polarización van hacia la estriola y en la sacular, en dirección opuesta. En la estriola abundan las células receptoras tipo I, caracterizadas por un cuerpo celular en forma de ánfora, envuelto 
por una dendrita aferente caliciforme (constituyen el $10-15 \%$ de toda la población celular). Estos receptores se diferencian morfológica y funcionalmente de los de tipo II, mucho más abundantes y situados en la periferia, de cuerpo cilíndrico y con múltiples botones sinápticos. Los aferentes de las células receptoras tipo I responden bruscamente a cambios en la aceleración lineal, jugando un papel principal en la "función dinámica» otolítica [4]; mientras que las aferencias sinápticas de las células tipo II tienen una respuesta mantenida a lo largo de la aceleración lineal («función estática»). Las fibras aferentes manifiestan una preferencia para fuerzas en una determinada dirección, reflejando la orientación de los receptores. Los aferentes utriculares son más específicos para fuerzas dirigidas horizontalmente en el plano de la mácula (es decir, para fuerzas laterales) y los aferentes saculares para fuerzas verticales.

El registro de las neuronas otolíticas aferentes primarias en el nervio vestibular del animal de experimentación, refleja las siguientes características:

- Tienen una tasa de descarga en reposo.

- Hay dos tipos de neuronas. Unas responden bruscamente a cambios en la aceleración lineal (de descarga irregular, procedentes de células receptoras tipo I) y otras mantienen una respuesta constante a lo largo de la aceleración lineal (descarga regular, procedentes de las células tipo II).

- Presentan un vector funcional de polarización. Esta sensibilidad bidireccional es asimétrica. En la dirección excitatoria la respuesta es mucho mayor que en la contraria.

Las neuronas de descarga regular muestran una escasa respuesta a los cambios en la aceleración lineal, pero la mantienen mientras dura. Sin embargo, los aferentes irregulares responden ante cualquier modificación de la aceleración lineal, pero se adaptan rápidamente cuando el estímulo se mantiene. La vibración por vía ósea (VO) provoca cambios bruscos en la aceleración lineal, consecuencia del movimiento que genera en el líquido endolinfático, de manera que al registrar las neuronas vestibulares primarias con una estimulación por VO de $500 \mathrm{~Hz}$ a escasa intensidad $(0,1 \mathrm{~g})$, se activa una gran proporción de neuronas otolíticas irregulares (utriculares y saculares) a nivel de la estriola. Sin embargo, las neuronas aferentes de los CS apenas reaccionan, salvo a muy elevadas intensidades (o a frecuencias más bajas, de $100 \mathrm{~Hz}$ ). La misma respuesta otolítica se puede desencadenar a través de un estímulo acústico por la vía aérea (VA), aunque a una intensidad mayor (120-130 dB SPL). En este caso, el movimiento del estribo sería el responsable de los desplazamientos ciliares en las células receptoras y por tal motivo, el sáculo (quizá por su mayor proximidad a la platina del estribo) tendría un umbral menor de respuesta [5]. En definitiva, tanto por VA como por VO se estimula a un grupo de aferencias vestibulares, que corresponden a las fibras irregulares del utrículo y del sáculo, procedentes de las células receptoras tipo I.

Conocer el recorrido de las neuronas otolíticas resulta fundamental para interpretar las pruebas clínicas (Figura 1). Los aferentes de la mácula utricular discurren por la división superior del nervio vestibular y la mayor parte de los aferentes saculares lo hacen por la rama inferior de este nervio. Hay una pequeña área rostral de la mácula (denominada «gancho de la mácula sacular»), cuyos aferentes forman el denominado nervio de Voit que camina por el nervio vestibular superior.

Estas neuronas aferentes primarias se proyectan sobre las de $2^{\circ}$ orden en el núcleo vestibular, donde se detecta un incremento de la tasa de descarga cuando se produce una inclinación de la cabeza hacia el mismo lado.

De manera similar a como ocurre con las proyecciones neuronales de los CS, existen unas fibras comisurales que contactan con el núcleo vestibular del lado opuesto y como luego veremos, las fibras maculares interaccionan con las de los CS. Las conexiones de los núcleos vestibulares a los núcleos de los motores oculares son poco conocidas y el sustrato neural de los movimientos compensatorios de posición, requeridos ante una aceleración lineal, están representados por las proyecciones otoliticoespinales, a través de los tractos vestíbuloespinales lateral y medial [1].

Los laberintos trabajan como un sistema globalmente equilibrado de manera que la excitación neural de un lado se acompaña invariablemente de la inhibición del otro [2]. El cerebro sintetiza la información de estos detectores para obtener un resumen final de dónde y cómo se mueve cada persona. Este funcionamiento global implica que la alteración de cualquiera de estos diez receptores sensoriales (tres CS y dos máculas de cada lado), produzca consecuencias en la integración vestibular general. 
A lo largo de los años, no se ha encontrado ningún procedimiento que permitiera evaluar clínicamente la función otolítica, porque provocar a un paciente una aceleración lineal de forma segura es difícil, peligroso y clínicamente impracticable. Para considerar la utilidad de cualquier prueba, esta debe ser segura, práctica, reproducible y además, específica y sensible a la disfunción otolítica [1].

La pérdida unilateral de la función otolítica estática es responsable de la «reacción de inclinación ocular» [6], en la que observamos que:

- Ambos ojos rotan alrededor del eje de visión hacia el lado afecto.

- La cabeza se inclina hacia el lado afecto.

- Hay una desviación oblicua entre los dos ojos, de manera que el ojo del lado de la lesión está más bajo que el del lado contrario.

Como consecuencia de estos cambios, la percepción visual se modifica y al pedirle al paciente que coloque en una habitación oscura una barra iluminada en posición vertical u horizontal, la inclina hacia el lado afecto. Esta prueba clínica se denomina, «prueba de percepción visual subjetiva de la vertical u horizontal» y se considera que desviaciones repetidas mayor de $2^{\circ}$ en pacientes con disfunción vestibular periférica, indicarían una alteración de la función otolítica estática [7].

EXPLORACIÓN OTOLITICA «DINÁMICA». POTENCIALES EVOCADOS MIOGÉNICOS VESTIBULARES (VEMPS)

Los trabajos iniciales del fisiólogo italiano Pietro Tullio (1881-1941), promovieron el estudio de la sensibilidad acústica del sistema vestibular [8], pero es a principios de los años 90, cuando Colebath y Halmagyi demostraron que era posible medir el potencial miogénico evocado en el músculo esternocleidomastoideo (ECM) a partir de una estimulación vestibular, dando nombre a los que desde entonces denominamos «Potenciales Evocados Miogénicos Vestibulares» (VEMPs: Vestibular Evoked Myogenic Potentials) [9], que provocaron la atención internacional [10]. Mediante electrodos de superficie dispuestos sobre la piel de determinados grupos musculares, se puede registrar una respuesta electromiográfica (EMG), que nos permite evaluar la función otolítica. Los estímulos desencadenantes fueron inicialmente estímulos acústicos por VA y más tarde, vibraciones por VO [11], cuya ventaja al saltar el oído medio consiste en evitar la influencia de una posible hipoacusia de conducción. Dado que el estímulo de $500 \mathrm{~Hz}$ (por VA y por VO), es muy específico para la activación de las neuronas otolíticas, es el que se utiliza habitualmente en clínica para obtener las respuestas miogénicas [1].

Desde sus comienzos se han descrito diferentes VEMPs en relación al grupo muscular activado por el input vestibular, pero los que tienen una base fisiológica más sólida, son los de origen cervical (denominados cVEMP) y los de origen ocular (oVEMP) [12], mucho más recientes [5], que se han utilizado como pruebas clínicas.

EI cVEMP se obtiene sobre el ECM contraído [13] y consiste en un potencial de corta latencia (13 mseg.), positivo (es decir, inhibitorio), denominado cVEMP p13 (primer pico con mayor positividad), n23 (el pico siguiente más negativo) [10]. Y el oVEMP es un pequeño potencial (5 a $10 \mu \mathrm{V}$ ), negativo (es decir, excitatorio), registrado mediante electrodos colocados debajo de los ojos, mientras el sujeto examinado mira hacia arriba.

Los parámetros de respuesta utilizados para describir e interpretar los VEMPs son la latencia de las ondas, su amplitud y el umbral de respuesta (que es el nivel más bajo de estimulación que permite detectar una onda) $[8,10]$. El valor absoluto de los VEMPs depende de muchas variables tales como la intensidad del estímulo, su frecuencia, la duración (más amplias con estímulos de unos $7 \mathrm{mseg}$ ) [14], la tensión muscular ejercida, la colocación de los electrodos, la transmisión del sonido (VA / VO), la edad, etc. [1]. La información diagnóstica procede de valorar la amplitud relativa de los potenciales registrados en un lado y otro, con estímulos simétricos. Aplicamos entonces un indicador denominado «razón de asimetría» $(A R)$, que se define de manera similar a la fórmula de Jongkees utilizada para valorar la asimetría calórica $[14,15]$ :

$$
A R=\frac{\text { VEMPizdo }- \text { VEMPdcho }}{\text { VEMPizdo }+ \text { VEMP dcho }} \times 100
$$

Donde «VEMP» se refiere a la amplitud del potencial. Un AR del $100 \%$ indicaría asimetría total y si no se supera el $40 \%$, se considera que 
la respuesta es simétrica (los valores medios varían del $7,2 \%$ al $23,1 \%$ ) [16].

\section{LOS VEMP CERVICALES (CVEMP)}

Las neuronas saculares estimuladas por un sonido, se continúan y hacen sinapsis con neuronas secundarias en el núcleo vestibular del mismo lado, que a su vez se proyectan ipsilateralmente a través del tracto vestibular medial en las motoneuronas espinales (del nervio espinal accesorio) ejerciendo una acción inhibitoria [17] (figura 1). Este cVEMP p13-n23 [9] es una respuesta saculocólica no cruzada, descendente e inhibitoria $[13,18,19]$.

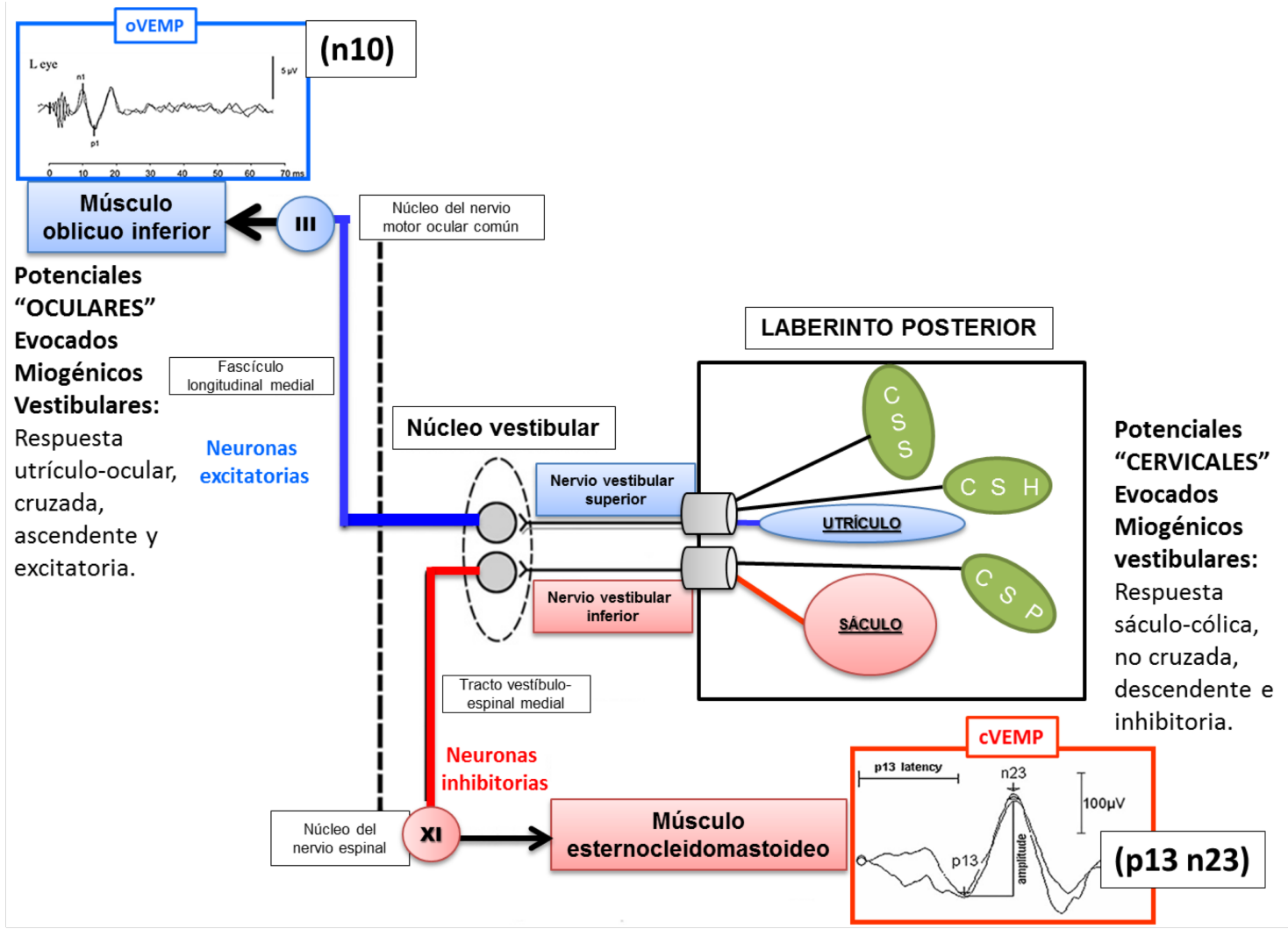

Figura 1. Vías nerviosas. En azul la vía utrículo-sacular (excitatoria y cruzada) y el potencial miogénico ocular (oVEMP) correspondiente y en rojo, la vía sáculo-cólica (inhibitoria y directa) con el potencial cervical (cVEMP). Modificado de Shin et al., 2012 [63].

LOS VEMP OCULARES (OVEMP) [5]

Un estímulo por VA o por VO desencadena debajo de los ojos un potencial negativo (excitatorio) de escasa amplitud y latencia (10 mseg) denominado oVEMP n10 [20]. Probablemente este potencial sea la respuesta miogénica del músculo oblicuo inferior, que es bilateral, pero más intensa debajo del ojo contralateral. Por tanto el oVEMP es una respuesta utrículo-ocular, cruzada, excitatoria y ascendente [19, 21] Figura 1). Se supone que los oVEMPs son una manifestación del reflejo vestíbulo-ocular (RVO) [5].S e ha comprobado que no son meros movimientos de los ojos pues están preservados en pacientes con exanteración ocular y conservación de la musculatura extraocular; y en casos de parálisis facial o de sordera profunda neurosensorial, por lo que ni son blink reflex, ni se afectan por el daño coclear [22].

\section{PROCEDIMIENTO DE REGISTRO DE LOS VEMPS} La gran variabilidad metodológica entre laboratorios, limita en ocasiones la comparación de resultados $[10,5]$, aunque cada uno debe obtener los suyos propios $[8,10]$. Sonidos «click» o «burst tonales» de $500 \mathrm{~Hz}$ intensos (100 dB $\mathrm{nHL}$ ), provocan un potencial inhibitorio amplio (60 a $300 \mu \mathrm{V}$ ), en el músculo ECM ipsilateral contraído. Es un potencial positivo-negativo, 
cuyos picos se encuentran a unos 13 y 23 mseg (p13-n23) [9] (Figura 2). Los componentes siguientes de la respuesta evocada, no tienen las propiedades del potencial p13-n23 y probablemente no dependan de los aferentes vestibulares.

A diferencia de los PEATC, que se generan por la descarga neuronal sincrónica, los cVEMPs se producen por descargas sincrónicas de unidades motoras, por lo que el potencial miogénico generado es de 60 a 800 veces mayor que el PEATC (de $200 \mu \mathrm{V}$ a menos de $1 \mu \mathrm{V}$ ). Cuando se utiliza un sonido por VA, realizaremos previamente una audiometría, pues la hipoacusia de transmisión disminuye la respuesta, al atenuar la intensidad del estímulo [23].

Si utilizamos la VO, el estímulo se calibra en dB FL (nivel de fuerza), donde la referencia es $1 \mu \mathrm{N}$ [10].

\section{REGISTRO CVEMP}

Para la evaluación clínica obtendremos 2-3 registros (las ondas deben ser replicadas) de 64 a 256 estímulos cada uno, a una intensidad de $100 \mathrm{~dB} \mathrm{nHL}$ (equivalente a $130 \mathrm{~dB} \mathrm{SPL}$ ) por $\mathrm{VA}$, generalmente a través de un auricular de inserción, colocado en el oído ipsilateral al ECM contraído. Se envía entonces un «click» (100 $\mu \mathrm{seg})$ o «burst tonal» de $500 \mathrm{~Hz}$ (6 a 8 mseg de duración [14], porque si se incrementa la duración, disminuye la amplitud, posiblemente por estimulación del músculo del estribo [8]). La frecuencia de estimulación suele ser de $5 \mathrm{~Hz}$ (5 veces por seg). El estímulo por VA de $500 \mathrm{~Hz}$ (rango 400-800 Hz [8, 14]) es con frecuencia más efectivo que el «click» [24]. La respuesta con «burst tonal» se reduce por VA a partir de $2 \mathrm{kHz}$. Se aplican tiempos de ascenso y descenso del estímulo, próximos a cero. Generalmente el paciente está sentado, con el cuello girado hacia el oído contrario al que se examina (también puede estar tumbado, girando la cabeza o elevándola). Se deben ofrecer periodos de descanso y el temblor del cuello indicaría fatiga. La mayoría tolera bien la exploración, pero los que presenten una movilidad cervical limitada o enfermedades neuromusculares que afecten al ECM, no son capaces de cooperar. La prueba tampoco puede realizarse si el paciente se encuentra en estado de inconsciencia. En sujetos con hiperacusia los estímulos intensos por VA podrían ser rechazados [10], precisándose la VO, mejor tolerada (en este caso, la respuesta óptima se obtiene con «burst tonales» de 200-
$250 \mathrm{~Hz}$, estando ausente con frecuencias superiores a $1 \mathrm{kHz}$ ).

Cada potencial se debe expresar en relación al nivel medio de contracción del EMG (que se debe monitorizar) [25], a fin de que la razón obtenida (Amplitud = P1 - N1 / EMG rms) sea función de la actividad muscular [8]. Es deseable que el grado de tonicidad del ECM (EMG rms del ECM), se mantenga de forma constante en sujetos adultos entre 50 y $200 \mu \mathrm{V}$ (en niños, más baja) [10].

El electrodo activo (+) no invertido, se coloca en el tercio medio del ECM [26], el de referencia (-) o invertido en la articulación esternoclavicular y el común o de tierra en la frente [10]. El rechazo de artefactos se puede poner en «off» pues la amplitud de los VEMPs es muy superior a la de los PEATC [10]. Como la energía dominante de la señal EMG está en el rango de 40 a $150 \mathrm{~Hz}$, la respuesta es filtrada con un filtro de paso alto entre 5 y $30 \mathrm{~Hz}$ y otro de paso bajo entre 1 y $2 \mathrm{kHz}[8,10]$; lo importante es definir los filtros que se usan y no modificarlos durante el estudio, porque podrían afectar la forma de las ondas [10]. Para obtener cVEMPs, la ganancia habitual es entre 2500 y 5000 (200-400 $\mu \mathrm{V} / \mathrm{V})$ [10]. La tasa de adquisición de muestras varía de 1 a $96 \mathrm{kHz}$, pero la mayoría usa entre 2,5 y $40 \mathrm{kHz}$ siempre asegurándose de que esta tasa sea de al menos 2 veces el valor del filtro de paso bajo [10]. Pequeñas desigualdades en latencia de un lado a otro, pueden deberse a la colocación de los electrodos sobre el músculo o a diferencias anatómicas musculares. La amplitud de los VEMP decae después de los 60 años [27].

\section{REGISTRO OVEMP}

El paciente puede estar tumbado, apoyando la cabeza en una almohada, manteniendo una discreta flexión. Tras limpiar la piel con alcohol se disponen los electrodos debajo de los ojos. El electrodo activo (+) en el reborde infraorbitario, $1 \mathrm{~cm}$ inferior al párpado y el de referencia -o indiferente (-)—, $2 \mathrm{~cm}$ por debajo del precedente; ambos alineados con el centro de la pupila. El de tierra se puede colocar en la frente, el mentón o el esternón [13]. Como en el registro cVEMP se intenta mantener una impedancia inferior a 5 kohms. El sujeto a explorar deberá mantener los músculos de sus mejillas relajados y mirar en la línea media y hacia arriba a una diana situada $30^{\circ}$ y $1 \mathrm{~m}$ por encima del eje visual de la mirada al frente, evitando en lo posible el parpadeo. Al mirar hacia arriba, el músculo oblicuo inferior se aproxima 
a la piel e incrementa su tonicidad al máximo [13]. El sujeto debe evitar elevar la cabeza y también lateralizar la mirada, que haría que los oVEMPs no fueran simétricos. El procedimiento es corto y poco molesto, pero requiere colaboración [28].

Los parámetros de estimulación son similares a los utilizados en cVEMPs [5]. Dado que los oVEMPs por VA se evocan mejor con frecuencias entre 400-1000 Hz, el «burst tonal» de 500 $\mathrm{Hz}$ es más específico que el «click», que tiene una banda más ancha [5]. Por VA el estímulo debe ser intenso (130 dB SPL o $100 \mathrm{~dB} \mathrm{nHL}$ ). La vibración ósea se sitúa en el punto $\mathrm{Fz}$ (punto medio de la frente en la unión con la línea del pelo). De esta manera se consigue una respuesta simétrica en ambos oídos, que no sería posible si se estimula a cada mastoides por separado. Aunque por VO se puede usar un «burst tonal» de $500 \mathrm{~Hz}$ (rango 100-750 $\mathrm{Hz}$ ), la máxima amplitud se obtiene con estímulos de alrededor de $100 \mathrm{~Hz}$ [5], que se emite en un periodo de $7 \mathrm{mseg}$, con un ancho de banda de 0,5-500 Hz, a una tasa de 3-5 / seg. y un período de ascenso lo más breve posible (idealmente cero). La intensidad máxima utilizada por VO es de $150 \mathrm{~dB}$ FL (= 31,6 N) [10]. El potencial de superficie obtenido, al ser más pequeño, se amplifica más que cVEMP (50.000 - 100.000). La actividad eléctrica pasa a través de unos filtros (1-10 Hz y $1 \mathrm{kHz})$. Se recogen de 100 a 200 estímulos para asegurar una reproductibilidad suficiente [13]. Las señales son promediadas (cada 30 a 50 presentaciones) con una tasa de muestreo de $20 \mathrm{kHz}$. El tamaño del oVEMP depende de lo bien que el paciente coloque su mirada, pero es variable de unos a otros, posiblemente en función del tamaño y la masa del cráneo (por VO). Sin embargo, la amplitud del oVEMP debajo de cada ojo es similar en el mismo sujeto [19]. Algunos pacientes no tienen oVEMP por VA, que es un estímulo más vulnerable que la $\mathrm{VO}$, por lo que éste se prefiere para la evaluación clínica.

La VO aplicada en la mastoides, ofrece oVEMPs de latencias más cortas y con mayor amplitud, pero donde no es posible la comparación con el otro oído [29].

Los oVEMPs decrecen en amplitud a partir de los 50 años [30]. Los oVEMPs por VO sólo se generan en el $50 \%$ de las personas mayores de 60 años. Sin embargo en niños a partir de los 3 años, no muestran diferencias en amplitud y latencia respecto al adulto.

\section{INTERPRETACIÓN DE LOS VEMPS}

Los VEMPs son pruebas vestibulares porque pacientes totalmente sordos, que no pueden oír el estímulo, muestran estos potenciales miogénicos por VA y VO [9]. Aunque los cVEMPs pueden estar ausentes en algunos casos con hipoacusia neurosensorial en tonos graves, como en la enfermedad de Ménière o en la sordera brusca [24], esta ausencia sugiere que el sáculo o el nervio vestibular inferior podrían estar involucrados [10].

Por el contrario, pacientes evaluados después de tratamiento con gentamicina sistémica, con ausencia de función vestibular bilateral, pero con audición residual, no tienen potenciales miogénicos [21]. La pérdida vestibular completa unilateral, provoca una reducción o ausencia de oVEMP n10 debajo del ojo contralateral y una reducción o ausencia de cVEMP p13- n23 sobre el ECM ipsilateral tanto con estimulación por VA como por VO.

A la vista de estas evidencias Curthoys [31] expuso la hipótesis de que las respuestas óculomotoras evalúan predominantemente la función utricular, mientras que las respuestas medidas en los músculos del cuello, valoran la función sacular (figura 1). Por tanto, es posible estudiar la función utricular y sacular separadamente, porque presentan proyecciones neuronales diferentes [32-34] y no, como se pensaba hasta ahora, porque el estímulo (por VA o VO) sea específico de una determinada mácula (ya que cada una responde tanto a estímulos sonoros como vibratorios). Una manera de confirmar esta hipótesis procede de los patrones de respuesta de los pacientes con neuronitis vestibular (NV), donde puede estar afectado todo el nervio o sólo su rama superior o la inferior. Los pacientes con neuritis vestibular superior (NVS) tienen cVEMPs normales (porque los aferentes saculares circulan a través del nervio vestibular inferior) y oVEMP n10 reducidos o ausentes por debajo del ojo contralateral. Y por el contrario, los pacientes con neuritis del nervio vestibular inferior (NVI), muestran cVEMP ipsilaterales reducidos o ausentes y potenciales oVEMP contralaterales normales (ver más adelante).

\section{APLICACIONES CLÍNICAS DE LOS VEMPS}

Como otras pruebas vestibulares, los VEMPs pueden utilizarse clínicamente para realizar el diagnóstico, conocer la extensión de una lesión o monitorizar la recuperación o progresión de una enfermedad. Una de sus ventajas (por ejemplo, en relación a la vertical / horizontal 
subjetiva) es que el reflejo permanece anormal, incluso después de la compensación central [28]. La sistemática de interpretación de las pruebas es común para cVEMPs y oVEMPs: la ausencia o atenuación de la amplitud de un reflejo, sugiere pérdida o disminución de función (salvo en los infrecuentes casos de hiperfunción vestibular), pero no indica la causa específica; mientras que un reflejo anormalmente amplio indicaría la presencia de una tercera ventana móvil en el laberinto (como una dehiscencia del CSS) $[8,28]$. Un alargamiento de la latencia con amplitud normal es poco frecuente y se suele asociar a anomalías de la conducción nerviosa a nivel del SNC.

\section{HaLlazgos CON VEMPS EN PATOLOgías VESTIBULARES TÍPICAS}

A continuación, exponemos un resumen de los hallazgos publicados con los VEMPs en relación a diferentes patologías vestibulares típicas.

\section{DEHISCENCIA DEL CSS (DCS)}

La presencia de una tercera ventana en el hueso laberíntico, permite que el sistema vestibular se active por el sonido (síntomas de vértigo, nistagmo, oscilopsia con o sin desequilibrio postural en respuesta a un sonido intenso es lo que se conoce como "fenómeno de Tu\|lio» [8]). Pero además de un nistagmo vestibular inducido por el sonido o la presión, numerosos estudios demuestran [8], que los VEMPs (cVEMP y oVEMP) de estos pacientes son de mayor amplitud y con niveles inferiores de umbral, tanto con estímulos por VA como por VO [28], presentando una sensibilidad y especificidad diagnóstica cerca del 90\% [35]. Resultados similares se han observado en pacientes con dilatación del acueducto vestibular [8]. Los oVEMP por VA son los que ofrecen la mejor discriminación, entre individuos normales y con DCS (con un incremento de la amplitud $\mathrm{n} 10$, hasta de 10 veces) [36]. Además, se altera la sintonización y pueden obtenerse potenciales con frecuencias más agudas de las habituales [37]. Normalmente, la respuesta óptima para los VEMPs por VA es entre $400 \mathrm{y}$ $1000 \mathrm{~Hz}$ y por VO entre 100 y $250 \mathrm{~Hz}$. En caso de DCS se registran por encima de $2 \mathrm{kHz}$, pudiendo utilizarse como herramienta diagnóstica (por ejemplo, obtención de potenciales a $4 \mathrm{kHz}$ ) [38]. Tras el cierre quirúrgico de la dehiscencia, los VEMPs se normalizan. Los oVE-
MPs también pueden ser más amplios en pacientes sintomáticos con «casi dehiscencia» del CSS [39].

Por tanto, los VEMPs permiten diferenciar una hipoacusia de transmisión causada por una DCS o por un acueducto vestibular ensanchado (VEMPs presentes de mayor amplitud), de la ocasionada por un proceso de oído medio (ausencia o reducción de VEMPs) [40].

\section{ENFERMEDAD DE MÉNIĖRE (EM)}

Los hallazgos son variables en función de los estudios [8]. Los VEMPs pueden ser normales en el 46-100\% de pacientes [8] o anómalos (de escasa o de gran amplitud, sobre todo al comienzo de la EM [41]). Las pruebas más sensibles serían los VEMPs por VA, más que por VO [42]. Los cVEMPs por VA se deteriorarían en fase inicial y posteriormente se alterarían las pruebas calóricas y los oVEMPs (la amplitud de oVEMP en el oído afecto, parece que se incrementa selectivamente durante las crisis en pacientes con EM reciente) [43]. De acuerdo a la anatomía del laberinto, la EM podría afectar inicialmente a la parte más inferior (cóclea y sáculo), para ascender posteriormente hacia el utrículo y los CS [44]. También es posible que se produzca un cambio de sintonización, de manera que la mejor respuesta con VEMP por VA no sea a $500 \mathrm{~Hz}$ sino a 1 $\mathrm{kHz}$ [45], sugiriéndose incluso, que la relación de amplitudes entre 500 / $1000 \mathrm{~Hz}$ sería un fiel indicador de la enfermedad. En definitiva, los diversos hallazgos podrían deberse a diferentes estadios del proceso, a la influencia de las crisis agudas y a la actividad fluctuante de la enfermedad [5].

Otra aplicación de los VEMPs en la EM, consiste en monitorizar el tratamiento con gentamicina intratimpánica [46].

La disfunción utricular ha sido implicada en los pacientes que tienen crisis de Tumarkin. En estos casos y en los que refieren sensación episódica de inclinación lateral, se ha observado una alteración en los oVEMP [47]. Basándose en las medidas de oVEMP de estos pacientes, parece que la función utricular fluctúa a lo largo del tiempo, recuperándose en unos días.

\section{NEURONITIS VESTIBULAR}

Generalmente afecta de forma selectiva al nervio vestibular superior (NVS), por lo que estos pacientes tienen reducción o ausencia del RVO procedente de los CSS y CSH, tanto con 
la prueba impulsiva cefálica (vHIT) como con la prueba calórica; y función utricular alterada, que se verifica a través de unos oVEMPs reducidos o ausentes en respuesta a una estimulación por VA o VO, preservándose la función sacular, con cVEMP normales. En los infrecuentes casos de NVI, se conserva la función del CSS, CSH y utrículo, pero está reducida o ausente la del CSP y sáculo, lo que se comprueba por la alteración de los impulsos cefálicos hacia el CSP y la falta de cVEMPs [48]. En un reciente trabajo prospectivo, el $55 \%$ de pacientes con neuronitis vestibular tienen alteradas ambas porciones del nervio, el $40 \%$ manifiesta NVS y tan sólo el 5\% NVI [49]. Los déficits sin embargo pueden ser desiguales y estar restringido el daño a áreas aisladas del oído interno (con afectación por ejemplo, del CSS y CSH pero con oVEMPs normales) [28]. Otro estudio de 133 pacientes con NV, concluye que la razón de asimetría en la amplitud de oVEMP determinaba el lado afecto con una sensibilidad del $90 \%$, especificidad de $80 \%$ y precisión diagnóstica del 94\% [50]. Después de una NV, tanto los cVEMPs como los oVEMPs pueden recuperarse en un $30 \%$ de pacientes entre 6 meses y 2 años [51] (la recuperación de oVEMP sería de buen pronóstico [49]).

Algunos pacientes con sordera brusca sufren vértigo, lo que sugiere la posibilidad de afectación de la rama inferior del nervio vestibular, al estar conectada por una anastomosis con el nervio coclear. En estos casos, los cVEMPs estarían más frecuentemente alterados que los oVEMPs [52].

\section{VÉRtigo Posicional Paroxístico Benigno} (VPPB)

En contraste con los que desarrollan VPPB después de una neuritis vestibular que generalmente conservan VEMPs normales, los pacientes con VPPB idiopático del CSP, especialmente si es recurrente, tienen mayor incidencia de anomalías en los oVEMPs (función utricular) que en los cVEMPs [28].

\section{INTERACCIÓN CS-MÁCULA}

Ante un movimiento centrífugo en el que se combinan la aceleración angular y la lineal, se demuestra que la aceleración lineal modula la velocidad lenta del nistagmo horizontal [53]. Las máculas otolíticas inhiben los CS, de manera que la sección aislada del nervio utricular provoca un nistagmo posicional horizontal [54]. En el curso de una NV el paciente se acuesta sobre el lado sano, porque sufre la inhibición del utrículo y disminuye la asimetría. Si se tumba sobre el enfermo, no se produce esta inhibición. Esta constatación es un argumento suplementario en favor del origen periférico del síndrome vestibular [55].

\section{VÉRTIGO E IMPLANTE COCLEAR (IC)}

La afectación vestibular es una posible complicación del IC, siendo frecuente observar una disminución de la respuesta calórica. También se ha observado una pérdida de la función sacular con cVEMP [56].

\section{SCHWANNOMA VESTIBULAR}

Aunque la mayor parte de los pacientes presentan hipoacusia unilateral, algunos sólo manifiestan ataxia vestibular, lo que no es sorprendente, pues la mayoría de estos tumores benignos nacen del nervio vestibular, generalmente del inferior.Los cVEMPs están alterados (retrasados, de escasa amplitud o ausentes) en 4 de cada 5 pacientes con neurinoma del acústico [57]. La latencia suele ser normal en el $90 \%$ de casos y el incremento se achacaría a la compresión sobre el TE o a la desmielinización que el tumor provoca sobre el nervio vestibular inferior. Como los cVEMPs no dependen ni de la función coclear ni de la del $\mathrm{CSH}$, pueden ser anómalos incluso cuando las pruebas calóricas resulten simétricas o los PEATC normales [8]. Los oVEMPs también se encuentran reducidos o ausentes.

ALTERACIONES DEL TRONCO DEL ENCÉFALO (TE) Lesiones unilaterales y focales, causadas por ictus o esclerosis en placas, especialmente las que afectan al núcleo vestibular, el núcleo del nervio accesorio y el tracto del vestíbulo espinal medial o su componente ascendente, el fascículo longitudinal medial, pueden alterar los cVEMPs ipsilaterales o los oVEMPs contralaterales. En la esclerosis múltiple y oftalmoplejia internuclear los oVEMPs se afectan con mayor frecuencia que los cVEMPs [5]. Los VEMPs son anómalos en el $30-80 \%$ de pacientes con esclerosis múltiple, siendo el retraso de latencia la principal anomalía y con menos frecuencia el descenso de amplitud. Estas alteraciones son más prevalentes en función de la evolución de la enfermedad [58]. Las lesiones cerebelosas preservan los VEMPs, hasta que se afecta el TE [59]. 
EVALUACIÓN DE LA FUNCIÓN OTOLÍTICA EN NIÑOS Hay pocas publicaciones en niños con resultados consistentes y por tanto hoy por hoy, no se puede dar una guía basada en la experiencia respecto a sus posibles aplicaciones [10]. Los oVEMPs tendrían la ventaja en niños pequeños que, si se utiliza una vibración ósea a tasa elevada (23 / seg), se podría completar la exploración en un menor tiempo, evitando la prueba térmica, tan molesta a esta edad.

\section{OTRAS CONDICIONES}

En aquellos pacientes con síntomas de vestibulopatía uni o bilateral (vértigo y ataxia) donde la exploración calórica e impulsiva es normal $(15 \%)$, los VEMPs pueden resultar inequívocamente anómalos [60]. Hay síntomas vestibulares característicos de afectación macular, como el "drop attacks», o las sensaciones de inclinación o translación, pero en general, cuando exista desequilibrio postural, se debe pensar en afectación otolítica [61]. En algunos pacientes con síndrome de Ramsay Hunt, se puede demostrar la ausencia de VEMPs por asociar afectación vestibular. $Y$ finalmente, en algún caso de hipoacusia neurosensorial unilateral por estrechamiento del conducto auditivo interno, se ha comprobado la persistencia de los VEMPs [8].

\section{CONCLUSIONES}

Las fibras aferentes utriculares y saculares se activan tanto por un estímulo acústico enviado por VA como por la vibración transmitida a través de la $\mathrm{VO}$, a consecuencia del movimiento generado en el líquido endolinfático. Mediante el registro de oVEMPs y cVEMPs podemos obtener de forma práctica, información clínica sobre las funciones utricular y sacular. Ambos procedimientos son específicos, sencillos, rápidos, reproducibles y seguros. Cuando estos resultados se combinan con otras pruebas vestibulares, se consigue llegar a tener un conocimiento funcional de cada uno de los receptores sensoriales del laberinto, que pueden verse afectados de manera independiente, provocando una sintomatología incapacitante [62].

\section{AGRADECIMIENTOS}

Al revisor de este trabajo, buen conocedor de la electrofisiología audiovestibular que con su esfuerzo ayudó a mejorar el contenido de este manuscrito.

\section{BIBLIOGRAFÍA}

1. Halmagyi GM, Curthoys IS: Otolith function tests. En: Herdmann SJ, Clendaniel RA: Vestibular Rehabilitation. F.A. Davis Company.

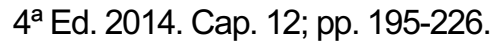

2. Halmagyi GM, Curthoys IS: Clinical anatomy and physiology of the vestibular system. En: Bronstein AM: Vertigo and Imbalance. Oxford University Press. $1^{a}$ Ed. 2013. Cap. 8; pp. 7991.

3. Curthoys IS, Manzari L. Otolithic disease: clinical features and the role of vestibular evoked myogenic potentials. Semin Neurol. 2013;33(3):231-7.

4. Goldberg JM. Afferent diversity and the organization of central vestibular pathways. Exp Brain Res. 2000;130(3):277-97.

5. Kantner C, Gürkov R. Characteristics and clinical applications of ocular vestibular evoked myogenic potentials. Hear Res. 2012;294(12):55-63.

6. Halmagyi GM, Gresty MA, Gibson WP. Ocular tilt reaction with peripheral vestibular lesion. Ann Neurol. 1979;6(1):80-3.

7. Pérez-Fernández N, Cervera-Paz FJ: Otras exploraciones del sistema vestibular. En: Bartual-Pastor J, Pérez-Fernández N: El sistema vestibular y sus alteraciones. Tomo I: Fundamentos y Semiología. Masson, S.A. $1^{\text {a }}$ Ed. 1998. Cap 15; pp. 239-255.

8. Akin FW, Murnane OD: Vestibular Evoked Myogenic Potentials. En: Jacobson GP, Shepard NT: Balance function assessment and management. Plural Publishing, Inc. $1^{\text {a }}$ Ed. 2008. Cap. 18; pp. 405-434.

9. Colebatch JG, Halmagyi GM. Vestibular evoked potentials in human neck muscles before and after unilateral vestibular deafferentation. Neurology. 1992;42:1635-6.

10. Papathanasiou ES, Murofushi T, Akin FW, Colebatch JG. International guidelines for the clinical application of cervical vestibular evoked myogenic potentials: an expert consensus report. Clin Neurophysiol. 2014;125(4):658-66.

11. Sheykholeslami K, Murofushi T, Kermany MH, Kaga K. Bone-conducted evoked myogenic potentials from the sternocleidomastoid muscle. Acta Otolaryngol. 2000;120(6):731-4. 
12. Todd NP, Rosengren SM, Aw ST, Colebatch JG. Ocular vestibular evoked myogenic potentials (OVEMPs) produced by air- and bone-conducted sound. Clin Neurophysiol. 2007;118(2):381-90.

13. Rosengren SM, Welgampola MS, Colebatch JG. Vestibular evoked myogenic potentials: past, present and future. Clin Neurophysiol. 2010;121(5):636-51.

14. Welgampola MS, Colebatch JG. Characteristics of tone burst-evoked myogenic potentials in the sternocleidomastoid muscles. Otol Neurotol. 2001;22(6):796-802.

15. Li MW, Houlden D, Tomlinson RD. Click evoked EMG responses in sternocleidomastoid muscles: characteristics in normal subjects. J Vestib Res. 1999;9(5):327-34.

16. Lee KJ, Kim MS, Son EJ, Lim HJ, Bang JH, Kang JG. The Usefulness of Rectified VEMP. Clin Exp Otorhinolaryngol. 2008;1(3):143-7.

17. Kushiro K, Zakir M, Ogawa Y, Sato H, Uchino Y. Saccular and utricular inputs to sternocleidomastoid motoneurons of decerebrate cats. Exp Brain Res. 1999;126(3):410-6.

18. Colebatch JG, Rothwell JC. Motor unit excitability changes mediating vestibulocollic reflexes in the sternocleidomastoid muscle. Clin Neurophysiol. 2004;115(11):2567-73.

19. Curthoys IS. The interpretation of clinical tests of peripheral vestibular function. Laryngoscope. 2012;122(6):1342-52.

20. Rosengren SM, McAngus Todd NP, Colebatch JG. Vestibular-evoked extraocular potentials produced by stimulation with boneconducted sound. Clin Neurophysiol. 2005;116(8):1938-48.

21. Iwasaki S, Smulders $Y E$, Burgess $A M$, McGarvie LA, Macdougall HG, Halmagyi $\mathrm{GM}$, et al. Ocular vestibular evoked myogenic potentials in response to bone-conducted vibration of the midline forehead at Fz. A new indicator of unilateral otolithic loss. Audiol Neurootol. 2008;13(6):396-404.

22. Chihara $\mathrm{Y}$, Iwasaki S, Ushio M, Fujimoto C, Kashio A, Kondo K, et al. Ocular vestibularevoked myogenic potentials (oVEMPs) require extraocular muscles but not facial or cochlear nerve activity. Clin Neurophysiol. 2009;120(3):581-7.
23. Bath AP, Harris N, McEwan J, Yardley MP. Effect of conductive hearing loss on the vestibulo-collic reflex. Clin Otolaryngol Allied Sci. 1999;24(3):181-3.

24. Hong SM, Byun JY, Park CH, Lee JH, Park MS, Cha Cl. Saccular damage in patients with idiopathic sudden sensorineural hearing loss without vertigo. Otolaryngol Head Neck Surg. 2008;139(4):541-5.

25. Welgampola MS, Colebatch JG. Vestibulospinal reflexes: quantitative effects of sensory feedback and postural task. Exp Brain Res. 2001;139(3):345-53.

26. Sheykholeslami K, Murofushi T, Kaga K. The effect of sternocleidomastoid electrode location on vestibular evoked myogenic potential. Auris Nasus Larynx. 200;28(1):41-3.

27. Welgampola MS, Colebatch JG. Vestibulocollic reflexes: normal values and the effect of age. Clin Neurophysiol. 2001;112(11):19719.

28. Weber KP, Rosengren SM. Clinical utility of ocular vestibular-evoked myogenic potentials (oVEMPs). Curr Neurol Neurosci Rep. 2015;15(5):22.

29. Tseng CC, Wang SJ, Young YH. Comparison of bone-conducted vibration for eliciting ocular vestibular-evoked myogenic potentials: forehead versus mastoid tapping. Otolaryngol Head Neck Surg. 2012;146(2):289-94.

30. Rosengren SM, Govender S, Colebatch JG. Ocular and cervical vestibular evoked myogenic potentials produced by air- and boneconducted stimuli: comparative properties and effects of age. Clin Neurophysiol. 2011;122(11):2282-9.

31. Curthoys IS. A critical review of the neurophysiological evidence underlying clinical vestibular testing using sound, vibration and galvanic stimuli. Clin Neurophysiol. 2010;121(2):132-44.

32. Kushiro K, Zakir M, Sato H, Ono S, Ogawa $Y$, Meng $\mathrm{H}$, et al. Saccular and utricular inputs to single vestibular neurons in cats. Exp Brain Res. 2000;13:406-15.

33. Uchino $Y$, Sato $H$, Sasaki $M$, Imagawa $M$, Ikegami $H$, Isu $N$, et al. Sacculocollic reflex arcs in cats. J Neurophysiol. 1997;77:300312. 
34. Uchino $\mathrm{Y}$, Sasaki $\mathrm{M}$, Sato $\mathrm{H}$, Imagawa $\mathrm{M}$, Suwa $\mathrm{H}$, Isu N. Utriculoocular reflex arc of the cat. $\mathrm{J}$ Neurophysiol. 1996;76:1896-903.

35. Brantberg K, Verrecchia L. Testing vestibular-evoked myogenic potentials with 90-dB clicks is effective in the diagnosis of superior canal dehiscence syndrome. Audiol Neurootol. 2009;14(1):54-8.

36. Janky KL, Nguyen KD, Welgampola M, Zuniga MG, Carey JP. Air-conducted oVEMPs provide the best separation between intact and superior canal dehiscent labyrinths. Otol Neurotol. 2013;34(1):127-34.

37. Taylor RL, Bradshaw AP, Halmagyi GM, Welgampola MS. Tuning characteristics of ocular and cervical vestibular evoked myogenic potentials in intact and dehiscent ears. Audiol Neurootol. 2012;17(4):207-18.

38. Manzari L, Burgess AM, McGarvie LA, Curthoys IS. An indicator of probable semicircular canal dehiscence: ocular vestibular evoked myogenic potentials to high frequencies. Otolaryngol Head Neck Surg. 2013;149(1):1425.

39. Ward BK, Wenzel A, Ritzl EK, Gutierrez-Hernandez S, Della Santina CC, Minor LB, et al. Near-dehiscence: clinical findings in patients with thin bone over the superior semicircular canal. Otol Neurotol. 2013;34(8):1421-8.

40. Minor LB. Clinical manifestations of superior semicircular canal dehiscence. Laryngoscope. 2005;115(10):1717-27.

41. Young $\mathrm{YH}, \mathrm{Wu} \mathrm{CC}, \mathrm{Wu} \mathrm{CH}$. Augmentation of vestibular evoked myogenic potentials: an indication for distended saccular hydrops. Laryngoscope. 2002;112(3):509-12.

42. Winters SM, Berg IT, Grolman W, Klis SF. Ocular vestibular evoked myogenic potentials: frequency tuning to air-conducted acoustic stimuli in healthy subjects and Ménière's disease. Audiol Neurootol. 2012;17(1):12-9.

43. Murofushi T, Nakahara H, Yoshimura E, Tsuda Y. Association of air-conducted sound oVEMP findings with cVEMP and caloric test findings in patients with unilateral peripheral vestibular disorders. Acta Otolaryngol. 2011;131(9):945-50.
44. Okuno T, Sando I. Localization, frequency, and severity of endolymphatic hydrops and the pathology of the labyrinthine membrane in Menière's disease. Ann Otol Rhinol Laryngol. 1987;96(4):438-45.

45. Node M, Seo T, Miyamoto A, Adachi A, Hashimoto $M$, Sakagami M. Frequency dynamics shift of vestibular evoked myogenic potentials in patients with endolymphatic hydrops. Otol Neurotol. 2005;26(6):1208-13.

46. Helling K, Schönfeld U, Clarke AH. Treatment of Ménière's disease by low-dosage intratympanic gentamicin application: effect on otolith function. Laryngoscope. 2007;117(12):2244-50.

47. Huang $\mathrm{CH}$, Young $\mathrm{YH}$. Ocular and cervical vestibular-evoked myogenic potentials in Tumarkin falls. Otol Neurotol. 2012;33(7):12516.

48. Manzari L, Burgess AM, Curthoys IS. Ocular and cervical vestibular evoked myogenic potentials in response to bone-conducted vibration in patients with probable inferior vestibular neuritis. J Laryngol Otol. 2012;126(7):68391.

49. Magliulo G, Gagliardi S, Ciniglio Appiani M, lannella G, Re M. Vestibular neurolabyrinthitis: a follow-up study with cervical and ocular vestibular evoked myogenic potentials and the video head impulse test. Ann Otol Rhinol Laryngol. 2014;123(3):162-73.

50. Manzari L, Tedesco A, Burgess AM, Curthoys IS. Ocular vestibular-evoked myogenic potentials to bone-conducted vibration in superior vestibular neuritis show utricular function. Otolaryngol Head Neck Surg. 2010;143(2):274-80.

51. Murofushi T, Iwasaki S, Ushio M. Recovery of vestibular evoked myogenic potentials after a vertigo attack due to vestibular neuritis. Acta Otolaryngol. 2006;126(4):364-7.

52. Fujimoto C, Egami N, Kinoshita M, Sugasawa K, Yamasoba T, Iwasaki S. Involvement of vestibular organs in idiopathic sudden hearing loss with vertigo: an analysis using oVEMP and cVEMP testing. Clin Neurophysiol. 2015;126(5):1033-8 
.53. Curthoys IS, Haslwanter T, Black RA, Burgess AM, Halmagyi GM, Topple AN, et al. Off-center yaw rotation: effect of naso-occipital linear acceleration on the nystagmus response of normal human subjects and patients after unilateral vestibular loss. Exp Brain Res. 1998;123(4):425-38.

54. Manzari L, Burgess AM, Curthoys IS. Does unilateral utricular dysfunction cause horizontal spontaneous nystagmus?. Eur Arch Otorhinolaryngol. 2012;269(11):2441-5.

55. Sauvage, JP. Vertiges: manuel de diagnostic et de réhabilitation. Elsevier Masson SAS. $1^{\text {a }}$ Ed. 2010. Cap. 4; pp. 23-32.

56. Krause E, Wechtenbruch J, Rader T, Gürkov $R$. Influence of cochlear implantation on sacculus function. Otolaryngol Head Neck Surg. 2009;140(1):108-113.

57. Patko T, Vidal PP, Vibert N, Tran Ba Huy P, de Waele C. Vestibular evoked myogenic potentials in patients suffering from an unilateral acoustic neuroma: a study of 170 patients. Clin Neurophysiol. 2003;114(7):1344-50.

58. Alpini D, Pugnetti L, Caputo D, Cesarani A. Vestibular evoked myogenic potentials in multiple sclerosis: a comparison between onset and definite cases. Int Tinnitus J. 2005;11(1):48-51
59. Su CH, Young YH. Differentiating cerebellar and brainstem lesions with ocular vestibularevoked myogenic potential test. Eur Arch Otorhinolaryngol. 2011;268(6):923-30.

60. Iwasaki S, Fujimoto C, Kinoshita M, Kamogashira T, Egami N, Yamasoba T. Clinical characteristics of patients with abnormal ocular/cervical vestibular evoked myogenic potentials in the presence of normal caloric responses. Ann Otol Rhinol Laryngol. 2015;124(6):45865.

61. Murofushi T, Komiyama S, Yoshimura E. Do patients who experience episodic tilting or translational sensations in the pitch plane have abnormal sacculo-collic reflexes?. Neurosci Lett. 2013;553:95-8.

62. Curthoys IS, Manzari L. Otolithic disease: clinical features and the role of vestibular evoked myogenic potentials. Semin Neurol. 2013;33(3):231-7.

63. Shin BS, Oh SY, Kim JS, Kim TW, Seo MW, Lee H, Park YA. Cervical and ocular vestibular-evoked myogenic potentials in acute vestibular neuritis. Clin Neurophysiol. 2012;123(2):369-75. 